\title{
Effect And Application Of Ionization Radiation (X-Ray) In Living Organism.
}

\author{
Suraj Raj Adhikari \\ Gogan Higher Secondary School, Lekhnath-13, Gagangauda, Kaski \\ Correspondence: adhikarisr@gmail.com
}

\begin{abstract}
$X$-ray is electromagnetic radiation of extremely short wavelength and high frequency. X-rays show wave as well as particle-like properties. X-ray is used in wide range of application such as industry, medicine and science application. It is essential tool in modern medicine. It can result in both beneficial and dangerous biological effects. These ionizing radiations penetrate the living tissues and can destroy living cells, chromosomal aberrations and carcinogenic effect.
\end{abstract}

Keywords: ionizing radiations, chromosomal aberrations, carcinogenic effect.

\section{Introduction}

$\mathrm{X}$-rays is a part of the electromagnetic energy traveling waves. X-rays are a form of ionizing radiations produced by accelerating or decelerating charged particles; examples include a beam of electrons striking a metal plate in an X-ray tube and a circulating beam of electrons in a synchrotron particle accelerator or storage ring. X rays were discovered accidentally in 1895 by the German physicist Wilhelm Conrad Roentgen while he was studying cathode rays in a high-voltage, gaseous-discharge tube. In addition, highly excited atoms can emit X-rays with discrete wavelengths characteristic of the energy level spacing in the atoms. The X-ray region of the electromagnetic spectrum falls far outside the range of visible wavelengths.

X-ray are electromagnetic radiation of extremely short wavelength and high frequency, with wavelengths ranging from about $10^{-8}$ to $10^{-12}$ meter and corresponding frequencies from about $10^{16}$ to $10^{20} \mathrm{~Hz}$.

\section{Wave Nature}

$\mathrm{X}$-rays exhibit wave as well as particle-like properties. $\mathrm{X}$-rays are a form of electromagnetic radiation; their basic physical properties are identical to those of the more familiar components of the electromagnetic spectrum-visible light, infrared radiation, and ultraviolet radiation. As with other forms of electromagnetic radiation, X-rays can be described as coupled waves of electric and magnetic fields traveling at the speed of light (about $300,000 \mathrm{~km} / \mathrm{sec}$ ). Their characteristic wavelengths and frequencies can be demonstrated and measured through the interference effects that result from the overlap of two or more waves in space. In 1906 the British physicist Charles Glover Barkla first demonstrated the wave nature of X-rays by showing that they can be "polarized" by scattering from a solid. Polarization refers to the orientation of the oscillations in a transverse wave; all electromagnetic waves are transverse oscillations of electric and magnetic fields.

X-rays also exhibit particle-like properties; they can be described as a flow of photons carrying discrete amounts of energy and momentum. This dual nature is a property of all forms of radiation and matter and is comprehensively described by the theory of quantum mechanics.

\section{Particle Nature}

In 1905 German physicist Albert Einstein had proposed that electromagnetic radiation is granular, consisting of quanta (later called photons) each with an energy $h f$, where $h$ is Planck's constant (about $6.6 \times 10^{-34}$ joule second) and $f$ is the frequency of the radiation. Einstein's hypothesis was strongly supported in subsequent studies of the photoelectric effect and by the successes of Danish physicist Niels Bohr's model of the hydrogen atom and its characteristic emission and absorption spectra. Further verification came in 1922 when American physicist Arthur Compton successfully treated the scattering of X-rays from the atoms in a solid as a set of collisions between X-ray photons and the loosely bound outer electrons of the 
atoms.

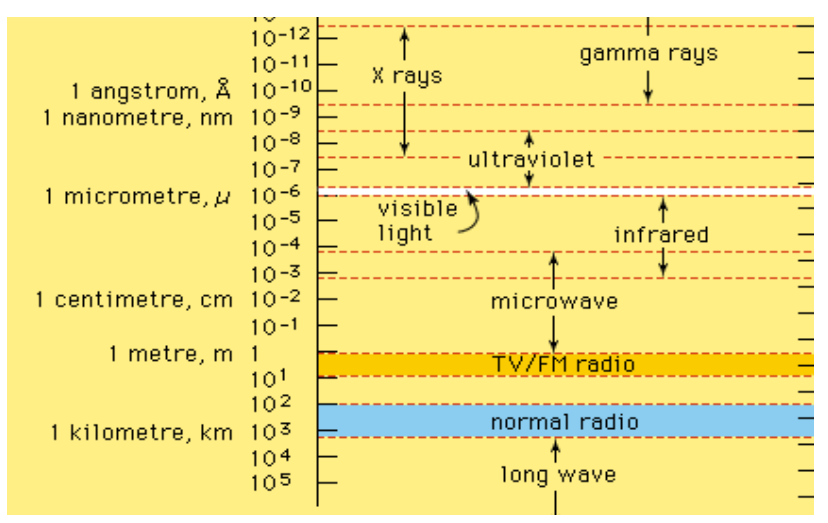

\section{Applications}

The ability of X-ray to penetrate optically opaque materials, their wavelengths of atomic dimension, the high energy of individual X-ray photons-lead to a wide range of industrial, medical, and scientific applications. Specialized X-ray sources, detectors, and analysis techniques have been developed to address a range of questions from the study of the interactions of the simplest molecules to the structure of the human brain.

X-ray images of the body are an essential diagnostic tool in modern medicine. Medical imaging allows for the non intrusive detection of dental cavities, bone fractures, foreign objects and diseased conditions such as cancer. In the 1970s a powerful new X-ray imaging technique, computed tomography (CT), was developed. Now in widespread use, CT scans produce detailed high-resolution cross-sectional images of internal organs and structures; they are far more sensitive to small density variations than conventional $\mathrm{X}$-ray images.

X-ray diffraction techniques (or "X-ray crystallography") allow for the determination of crystal structures in inorganic, organic, and biological materials. The detailed atomic structure of the doublehelix polymer deoxyribonucleic acid (DNA) was famously discovered by James Watson and Francis Crick via the X-ray crystallography studies of Maurice Wilkins. X-ray fluorescence is a complementary method for the quantitative analysis of the composition of materials. In this technique, a sample is exposed to either an electron beam or a beam of primary X-rays; the resulting atomic excitations lead to $\mathrm{X}$-ray emissions with wavelengths characteristic of the elements in the sample. X-ray fluorescence and diffraction techniques are valuable methods for the nondestructive analysis of art objects. Many of the above techniques are enhanced by the exceptionally high X-ray intensities. In agricultural industries the irradiation of some foods with X-rays and gamma rays is used to inhibit selectively the growth of bacteria.

Radiation can result in both beneficial and dangerous biological effects. As with other forms of ionizing radiation, X-rays cause biochemical changes in living cells. A high-energy X-ray photon deposits its energy by liberating electrons from atoms and molecules. These free electrons may themselves ionize additional neutral species. Through this process, reactive ions and free radicals are formed, leading to further chemical reactions. The resulting radiation-induced chemistry can break the molecular bonds needed for cell growth and can induce genetic damage. While there are significant health risks associated with exposure to $\mathrm{X}$-rays, radiation therapies exploit the above effects to treat cancerous tumors and blood disorders such as leukemia. X-rays (and higher-energy gamma rays) are directed at target tissues; the consequent molecular damage blocks the growth of the diseased cells.

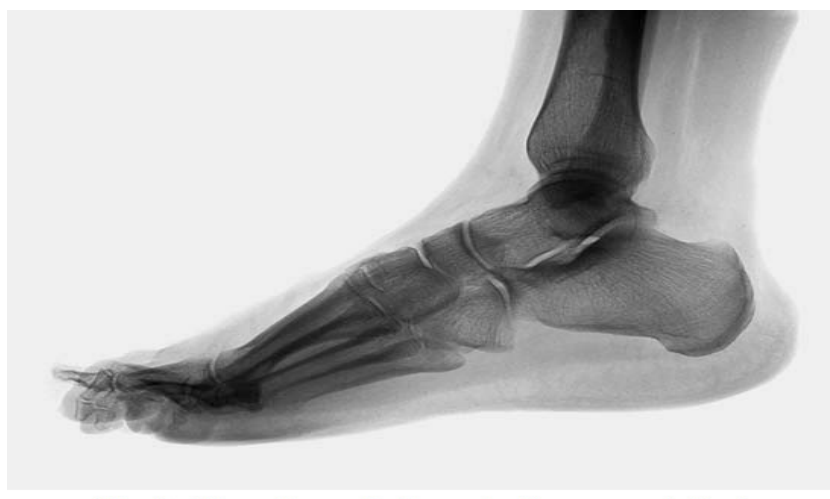

Fig: An X-ray shows the bones inside a person's foot.

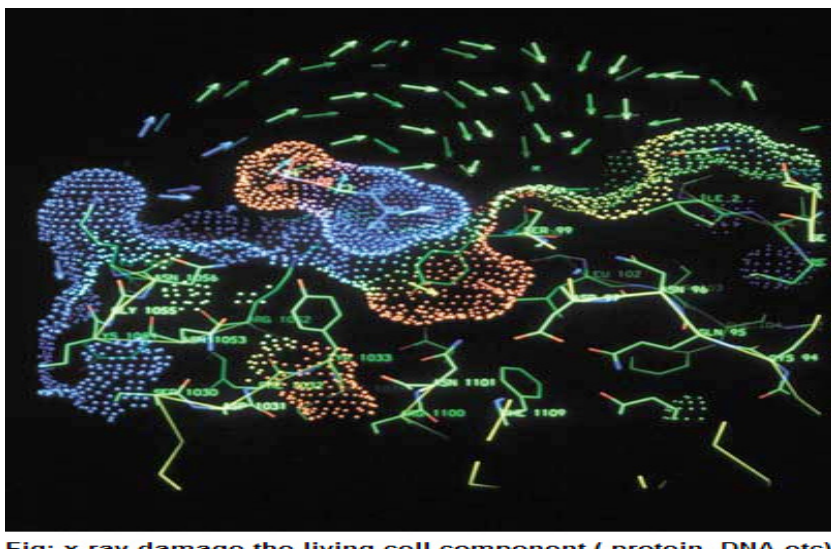




\section{Radiation injury}

Tissue damage or changes caused by exposure to ionizing radiation - namely, gamma rays, $\mathrm{X}$ rays, and such high-energy particles as neutrons, electrons, and positrons. Sources of ionizing radiation may be natural (e.g., radioactive substances such as the element radium or the radioisotopes potassium- 40 and carbon14) or man-made (X-ray machines, nuclear reactors, particle accelerators, nuclear weapons, etc.).

These ionizing radiations penetrate the living tissues and can destroy living cells or make them functionally abnormal (Nerilee Baker, et.al.1990). Ionizing radiations including alpha, beta and gamma rays and neutrons with sufficient energy to generate ion pairs i.e. electrons, which can generate chemically active free radicals these in turn can damage the molecular structure resulting in cell dysfunction (somatic effect) or mutations (genetic damage) (Masumura K, et.al. 2002). Recently National cancer institute (NCI) of USA considering adding medical X-rays to human carcinogenic list (Anonymous, 2003)

The hazards of exposure to ionizing radiation were recognized shortly after Roentgen's discovery of the $\mathrm{x}$-ray in 1895. Acute skin reactions were observed in many individuals working with early $\mathrm{x}$-ray generators, and by 1902 the first radiation-induced cancer was reported arising in an ulcerated area of the skin. Then, few years later, a large number of cancers were observed, in 1911 leukemia was diagnosed in five radiation workers. Indeed, Marie Curie and her daughter Irene are both thought to have died of radiation-induced leukemia. Since that time, so many experimental and epidemiological studies have confirmed the oncogenic effects of radiation in many tissues of many species (Kufe et.al. 2003). The vast majority of people are exposed to very low doses of radiation; this may contribute only for a small amount to the overall cancer burden. But radiation continues to be perceived by the public as a major carcinogen. This perception likely comes from images of wartime, uses of nuclear weapons and, more recently, reactor accidents such as Chernobyl (John et. al. 1996). Effect of X- and Gamma rays on DNA in Human Cells radiation can penetrate into living cells resulting in the transfer of radiation energy to the biological material. The absorbed energy can induce formation of reactive oxygen species, breaking of chemical bonds and ionization of different biologically essential macromolecules, such as DNA, membrane lipids and proteins. The DNA damage induced by radiation such as base alteration, cross linking, strands break or chromosomal aberration can lead to mutation (Ahmed 2005). Oxidation of DNA can result in damage to all four bases and to the deoxyribose. Among oxidative base damages in DNA, C8-oxidation form of guanine, 7, 8-dihydro-8-oxoguanine (8-oxoG) is one of the major base lesions and is often used as a key indicator of cellular oxidative stress. Oxidative damage to DNA can be induced exogenously by radiation or chemical agents or endogenously by free radicals released during respiration (Ishida 2002, Aust and Eveleigh 1999).

Ionizing radiation can randomly cause damage to all cellular components and induces a variety of DNA lesions, like single strand break, double strand break or base damage (Schmid,et. al, 2007). These many observations suggest that biological response to ionizing radiation is highly complex processes that depend on both dose and dose rate (Ogata et. al, 2005).

Radiation injury occurs in various forms, with each type dependent on the ionizing radiation involved, its penetrating ability, the portion of the body exposed, the duration of exposure, and the total dose. Radiation injury occurs most readily in tissues and organs consisting of rapidly proliferating cells; for example, the skin, the lining of the gastrointestinal tract, and the bone marrow, where progenitor cells multiply continuously to replace the mature cells that are constantly being lost through normal aging. The effects of radiation on these organs result primarily from the destruction of the progenitor cells and the consequent interference with the replacement of the mature cells, which is so vital to the maintenance of tissue structure and function.

Other manifestations of radiation injury are certain forms of cancer. The survivors of the atomic-bomb blasts at Hiroshima and Nagasaki, some patients subjected to multiple fluoroscopic chest examinations, and certain groups of radiation workers (e.g., women who painted radium watch and clock dials) have exhibited dose-dependent increases in the incidence of cancer, most notably leukemia and breast cancer.

Radiation injury also includes abnormalities produced 
in the embryo. The tissues of the embryo, like others composed of rapidly proliferating cells, are extremely sensitive to ionizing radiation. Organs irradiated during the process of formation thus tend to be malformed. A large number of these are malformations of the nervous system, such as reduced brain size or the failure of the eyes to develop. Nervous-system abnormalities in human infants have been found with higher-thannormal frequency among children born to women who were pregnant and living in Hiroshima and Nagasaki at the time of the atomic-bomb explosions. The incidence of mental retardation and reduced head size in such children increased substantially when exposure occurred between the 8th and 15th weeks of gestation, which has been determined to be the age of greatest susceptibility to ionizing radiation.

\section{Conclusion}

Ionization radiations are used in diverse modern application. It is used in diagnosis of disease, preserve of food material, purification of material and identification of component and compound. These ionizing radiations enter the living tissues and can destroy living cells, chromosomal aberrations and carcinogenic effect. Ionizing radiation can randomly cause damage to all cellular components and induces a variety of DNA effect. So x-ray is utilized in wellprevent and protective condition.

\section{References}

1. Ahmed R. G. (2005), Damage pattern as function of various types of radiations, Medical J. of Islamic World Academy of Science 15, 135-147.

2. Anonymous news (2003), Journal of nuclear medicine, 44 (2): 9N, 2003.

3. Aust A.E., Eveleigh J. F. (1999), Mechanisms of DNA oxidation, Proceedings of the Society for Experimental Biology and Medicine, USA, 222, 246-252.

4. Faraj et. al. (2011), Effect of X-and Gamma Rays on DNA in Human Cells, European Journal of Scientific Research. 53 (3), 470-476.

5. Ishida H. (2002), Molecular dynamics simulation of 7, 8-dihydro-8-oxoguanine DNA, Biomolecular Structure and Dynamics 19, 839-851.

6. John.D. B, Peter.D. I. Jr., "Leukemia", W.B.Saunders (1996), chapter 11, 195-209.

7. Kufe D., Pollock R., Weichselbaum R., Bast Jr R., Ganster T., J. Holland (2003)," Cancer Medcine “, BC Decker Inc. Hamilton, London .

8. Masumura K, Kuniya K, Kurobe T, Fukuoka M, Yatagai F, Nohmi T. et al. (2002), Ion-induced mutations in the gpt delta transgenic mouse: comparison of mutation spectra induced by heavyion, X-ray, and gamma-ray radiation, Environ Mol Mutagen; 40 (3): 207-15.

9. Nerilee Baker, Luke Bromilow and Joan Costigan, Exposure to ionizing - radiation from X-rays in the intensive therapy unit St. Vincent's Hospital. Nursing monograph. 1990; 1: 19-26.

10. Ogata H., Furukawa C., Kawakami Y., Magae J. (2005), A quantitative model for the evaluation of dose rates effects following exposure to low-dose gamma radiation, Radioprotection 40, 191- 202.

11. Schmid E., Schrader T. (2007), Different biological effectiveness of ionizing and nonionizing radiations in mammalian cells, Adv. Radio Sci. 5, 1-4.

12. www.wikipedia.com/x-ray 